\title{
Ventilatory Muscle Endurance Training in Quadriplegia: Effects on Breathing Pattern
}

\author{
B. Loveridge, ${ }^{1}$ BPT, MSc, PhD, M. Badour, BPT, H. Dubo, ${ }^{2}$ MD, \\ FRCPC \\ Spinal Cord Injury Unit \& Spinal Cord Research Centre, University of \\ Manitoba, and Health Sciences Centre, Winnipeg, Manitoba, Canada. ${ }^{1}$ School of \\ Medical Rehabilitation, ${ }^{2}$ Department of Medicine, University of Manitoba, \\ Winnipeg, Manitoba, Canada.
}

\section{Summary}

We examined the effects of ventilatory muscle endurance training on resting breathing pattern in 12 C6-C7 traumatic quadriplegics at least 1 year post-injury. All subjects had complete motor loss below the lesion level. Subjects were randomly assigned to a training $(N=6)$, or a control group $(N=6)$. Baseline tests included measurement of resting ventilation and breathing pattern using mercury in rubber strain gauges for 20 minutes in a seated position; maximum inspiratory mouth pressure $(M I P)$ at $F R C$, and sustainable inspiratory mouth pressure for 10 minutes $(S I P)$; lung volumes, and arterial blood gases ( $A B G$ 's). The training protocol consisted of breathing through an inspiratory resistor equivalent to $85 \%$ SIP for 15 minutes twice daily, 5 days a week for 8 weeks. Both trainers and controls attended the lab every 2 weeks for reassessment of MIP and SIP and the inspiratory resistance was increased in the training group as SIP increased. At the end of 8 weeks, baseline tests were repeated. All subjects had normal $A B G$ 's. There was a significant increase in mean MIP and SIP in both the control group $(30 \% \pm 19 \%$ and $31 \% \pm 18 \%$ respectively), and in the training group $(42 \% \pm 24 \%$ and $78 \% \pm 49 \%$ respectively). Although the absolute values for both MIP and SIP were greater in the training group than in the control group, the differences were not significant. The alterations in resting breathing pattern were also the same in both groups. Mean frequency decreased significantly in the control group (20.2/minute to $16.9 /$ minute) and, while insignificant, the change in frequency in the training group was the same, 19.4/minute to 16.4/minute. Mean tidal volume (Vt) increased $18.2 \%$ of baseline Vt in the control group and $17.0 \%$ baseline in the trainers, resulting in no change in minute ventilation. As MIP and SIP increased similarly in both groups, the data from the control and trainers was pooled and timing changes re-evaluated pre-and post-study. A significant decrease in mean Ti/Ttot was observed, while no change in Vt/Ti was found. We concluded that the testing procedure 
itself provided the stimulus resulting in a significant increase in MIP and SIP. The addition of training did not increase MIP and SIP further. The increased MIP and SIP resulted in a slower and deeper breathing pattern and a significantly shorter Ti/Ttot in both trainers and control subjects.

Key words: Paraplegia; Quadriplegia; Respiratory muscles; Exercise training; Breathing control

The breathing pattern of quadriplegics, as in other patients with respiratory muscle weakness, appears to be more rapid and shallow than in normals (Bergofsky, 1964). Maximum inspiratory mouth pressure (MIP), an index of ventilatory muscle strength, is often also markedly reduced as compared to normals (Gross et al., 1980; Leith and Bradley, 1976). It has been demonstrated that the strength and endurance of the ventilatory muscles can be improved through a programme of inspiratory resistive training in normal subjects (Leith and Bradley, 1976; Clanton et al., 1985a), chronic obstructive pulmonary disease (COPD) patients (Pardy et al., 1981; Belman and Mittman, 1980), as well as quadriplegics (Gross et al., 1980). Such training regimes have reported symptom relief and subjective improvements in breathing in quadriplegics (Gross et al., 1980), and increased exercise performance in COPD (Grassino et al., 1979). While such training regimes are thought to be important in protecting the ventilatory muscles against fatigue (Gross et al., 1980; Pardy et al., 1981; Grassino et al., 1979), little information is available concerning the effects such training may have on resting breathing pattern or breathing control. We hypothesised that increasing the strength and endurance of the ventilatory muscles would alter resting breathing pattern by increasing tidal volume $(\mathrm{Vt})$ and decreasing frequency $(F)$. Such a slower, deeper breathing pattern is more efficient and might lessen the adverse effects of chronic hypoventilation which may occur in this group.

\section{Methods}

Twelve quadriplegics with complete motor loss below the level of the sixth and seventh cervical vertebrae were recruited from the outpatient department of the Rehabilitation Hospital's Spinal Cord Unit. All subjects were at least 1 year post-injury and were medically stable at the time of entry into the study. Informed consent was obtained from all individuals. Subjects were randomly assigned to a control $(\mathrm{N}=6)$ and training group $(\mathrm{N}=6)$. The baseline characteristics of the subjects are listed in Table I. A pre-test questionnaire was completed with each subject to document drugs, activity level, symptoms with activity, and electric versus standard wheelchair. Baseline pulmonary function tests, lung volumes, and arterial blood gases (ABG's) were measured. Functional residual capacity (FRC) was determined using a closed circuit nitrogen washout technique. Total lung capacity (TLC) was calculated by adding inspiratory capacity (IC) to the FRC measurement, and residual volume (RV) was calculated by subtracting FVC from TLC.

Maximum inspiratory pressure (MIP) was assessed using a comparable technique to that described by Black and Hyatt (1971), using a $\pm 100 \mathrm{~cm} \mathrm{H}_{2} \mathrm{O}$ Vali- 
Table I Characteristics of quadriplegic subjects

\begin{tabular}{|c|c|c|c|c|c|}
\hline & $\begin{array}{l}\text { Level of } \\
\text { lesion }\end{array}$ & Age & $\begin{array}{l}\text { Height } \\
(\mathrm{cm})\end{array}$ & $\begin{array}{l}\text { FVC } \\
(0, \text { Pred })\end{array}$ & $\begin{array}{l}\mathrm{FEV}_{10} / \mathrm{FVC} \\
\left(\begin{array}{l}0 \\
0\end{array}\right)\end{array}$ \\
\hline \multicolumn{6}{|c|}{ Trainers } \\
\hline 1 & C6 & 27 & 175 & 47 & 100 \\
\hline 2 & C6 & 35 & 188 & 71 & 95 \\
\hline 3 & $\mathrm{C} 7$ & 28 & 180 & 85 & 86 \\
\hline 4 & C6 ACS & 28 & 173 & 46 & 92 \\
\hline 5 & C6 ACS & 31 & 164 & 85 & 69 \\
\hline 6 & C6 ACS & 37 & 178 & 41 & 89 \\
\hline$\overline{\mathrm{X}}$ & & 31 & 176 & 63 & \\
\hline S.D & & $4 \cdot 1$ & $7 \cdot 9$ & 20 & \\
\hline \multicolumn{6}{|c|}{ Controls } \\
\hline 1 & C6 & 49 & 175 & 60 & 100 \\
\hline 2 & $\mathrm{C} 7$ & 22 & 180 & 58 & 91 \\
\hline 3 & $\mathrm{C} 7$ & 28 & 175 & 60 & 86 \\
\hline 4 & C6 & 26 & 183 & 85 & 72 \\
\hline 5 & $\mathrm{C} 7$ & 49 & 188 & 114 & 83 \\
\hline 6 & C6 & 36 & 195 & 55 & 100 \\
\hline$\overline{\mathrm{X}}$ & & 35 & 183 & 72 & \\
\hline S.D. & & 12 & $7 \cdot 8$ & 23 & \\
\hline
\end{tabular}

ACS: Anterior Cord Syndrome.

dyne pressure transducer. Measurements were recorded at FRC. Subjects wore noseclips, and were instructed to breathe normally through a mouthpiece to establish FRC. Then at the end of a normal expiration subjects were instructed to breathe in against an occluded mouthpiece. Measurements were repeated a minimum of 10 times until 3 measurements within $5 \%$ of each other were recorded. The average of the 3 best measurements was calculated as MIP.

Sustainable inspiratory pressure (SIP) was used as an index of ventilatory muscle endurance. A modification of the technique described by Knickerson and Keens (1982) was employed which required that the quadriplegics only had to complete 2 endurance runs during each testing session. We built a similar threshold load device to that of Knickerson and Keens (1982) (Fig. 1). The relation between the weight applied to the threshold load device and the pressure that must be generated to produce airflow was linear, with an opening pressure of $-8 \mathrm{~cm} \mathrm{H}_{2} \mathrm{O}$. Once opened, flow was independent of pressure. The initial SIP test was conducted with the subject generating a pressure equivalent to $75 \%$ MIP. The subject was instructed to continue breathing against this load as long as possible. A single lead ECG and end-tidal $\mathrm{CO}_{2}$ (Perkin-Elmer Medical Gas Analyzer, model 1100) were monitored throughout the run. The patient was also instructed to come off the device if subjective symptoms of dizziness, lightheadedness, or undue fatigue were experienced. If the subject was able to breathe against the load for more than 10 minutes, the second test was performed with a load equivalent to $85 \%$ MIP. If the first run was less than 10 minutes, the load was decreased to $65 \%$ MIP for the second run. A rest period equal to at least 10 times the length of the preceeding test was provided between each run (Nickerson and Keens, 1982). A pressure/time plot was constructed and the pressure that the subject could breathe against for 10 minutes was extrapolated from these two points. This extrapolated pressure at 10 minutes was called SIP. 


\section{ENDURANCE TESTING RIG}

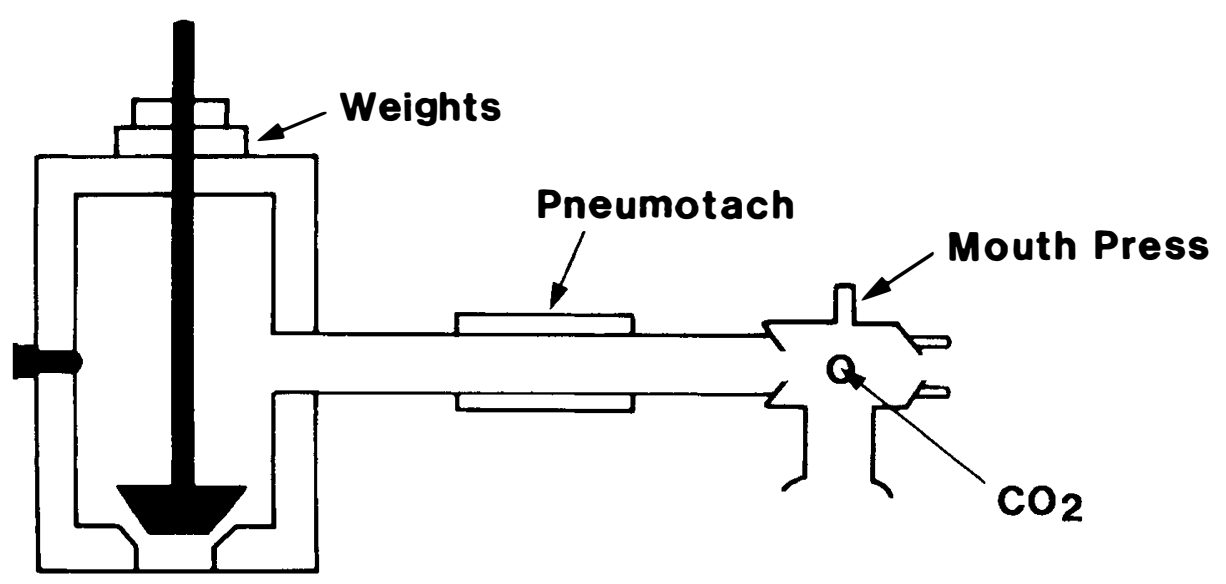

Figure 1 Ventilatory muscle endurance testing rig.

The trainers were provided with an inspiratory resistor that would require the generation of a pressure equivalent to $85 \%$ SIP at normal resting flow rates. The patient was instructed to breathe on this resistor while wearing nose clips, twice daily for 15 minutes, 5 days a week, for 8 weeks. Patients were allowed to set their own breathing rate and pattern during training and the training strategy employed was not monitored. Each patient was required to keep a daily diary to monitor performance. Both trainers and controls attended the lab every 2 weeks and repeated the tests for MIP and SIP. The inspiratory resistor was increased for the trainers as SIP increased.

Ventilation and breathing pattern were assessed using mercury in rubber strain gauges. Subjects were seated in their wheelchairs for a period of $20 \mathrm{~min}-$ utes, and a single lead electrocardiogram was also recorded during this period. Subjects watched a videotaped movie to distract them from the monitoring devices. They were instructed to sit still and to avoid arm or trunk movements, both of which introduce artifacts in the strain gauge signals.

The ribcage strain gauge ( $\mathrm{RC}$ ) was positioned approximately 1 inch below the nipple level on the chest wall, and the abdominal strain gauge (ABD) was placed half way between the umbilicus and the xiphoid process, around the upper abdomen. These positions provided the maximum signal for both strain gauges. A 3 inch wide and half inch thick foam pad was placed over the spinous processes posteriorly under the strain gauges to protect the patient from undue pressure in this region during the test. The output signals from both strain gauges were converted into a DC voltage and were sampled at $20 \mathrm{~Hz}$ by a Hewlett Packard microcomputer (Model 9816) and simultaneously recorded on a polygraph (Model 2600S, Gould Recorder).

The calibration technique has been previously described in detail using respiratory inductance plethysmography (RIP) (Loveridge et al., 1983), and strain gauges have been demonstrated to be equally as accurate as the RIP in normal 
Table II Pulmonary function and lung volume data pre- and post-training

\begin{tabular}{lcccc}
\hline & \multicolumn{2}{l}{ Trainers $(\mathbf{N}=6)$} & \multicolumn{2}{c}{ Controls $(\mathbf{N}=6)$} \\
$\%$ Pred & Pre & Post & Pre & Post \\
\hline TLC & $77 \cdot 3$ & 82 & $91 \cdot 6$ & $91 \cdot 6$ \\
& $\pm 9 \cdot 5$ & $\pm 6 \cdot 9$ & \pm 17 & \pm 17 \\
RV & 107 & 122 & $125 \cdot 5$ & 126 \\
& \pm 15 & \pm 24 & \pm 41 & \pm 38 \\
FRC & 78 & 83 & 82 & 79 \\
& \pm 11 & \pm 15 & \pm 23 & \pm 18 \\
IC & 80 & 95 & 9 & 104 \\
& \pm 12 & \pm 15 & \pm 25 & 75 \\
FVC & 63 & 64 & 72 & \pm 23 \\
& \pm 20 & \pm 10 & \pm 23 & \\
\hline
\end{tabular}

subjects using this calibration technique (Loveridge et al., 1984a). Briefly, the strain gauges were calibrated in the seated position and it's stability was checked at the end of the data acquisition period. During calibration, the subject wore noseclips and breathed through a pneumotachygraph for a period of 1 minute. The pneumotachygraph signal was integrated digitally. The analog signals from the $\mathrm{RC}, \mathrm{ABD}$, and the pneumotachygraph were sampled simultaneously at 20 $\mathrm{Hz}$ by a microcomputer. Only inspiratory data were analysed. Multiple linear regression analysis was used to determine $\mathrm{RC}$ and $\mathrm{ABD}$ calibration factors. The strain gauges were considered calibrated on the basis of the tightness of fit between the pneumotachygraph derived volume and the predicted volume from the summed RC and ABD signals. A summed RC and ABD volume less than $10 \%$ deviation from the pneumotachygraph measured volume was considered to be an acceptable calibration. At the end of the study, subjects again breathed through a pneumotachygraph for a 1 minute period and the accuracy of the RC and ABD calibration factors derived during the initial calibration were verified.

The best fit multiple linear regression equation from calibration was used to reconstruct a spirogram from the summed RC and ABD signals. Segments in which there were movement artifacts were identified from the chart recording and deleted from the computer data file prior to analysis. Subjects were indirectly observed through the test and any movement was noted on the chart recording. The peak (end of inspiration), and the trough (beginning of the next inspiration) were identified manually with the assistance of an $x-y$ plotter on the computer. These values were then stored by the computer and used to determine tidal volume $(\mathrm{Vt})$, inspiratory time $(\mathrm{Ti})$ and expiratory time $(\mathrm{Te})$. The remaining timing components of the spirogram were derived from these values. The mean, standard deviation and coefficient of variation of each breath component was calculated. A computer histogram program was used with the tidal volume data to identify sighs (breaths greater than $3 \times$ mean $\mathrm{Vt}$ ).

\section{Results}

Arterial blood gases were normal, and routine pulmonary function tests and lung volume measurements remained unchanged in both groups post-study (Table II). In 3 trainers with lower initial IC's (67-72\% predicted), there was substantial improvement in IC post-training. However, the other 3 trainers 
Table III Strength (MIP) and endurance (SIP) of the ventilatory muscles

\begin{tabular}{|c|c|c|c|c|c|c|}
\hline & $\begin{array}{l}\text { Streng } \\
(-\mathrm{CM}\end{array}$ & Press) & & $\begin{array}{l}\text { Endura } \\
\text { (-CM }\end{array}$ & Press) & \\
\hline & Pre & Post & ${ }^{\circ}$ o Increase & Pre & Post & ${ }_{0}$ Increase \\
\hline Controls & $81 \cdot 7$ & $105 \cdot 3^{\star}$ & $30 \cdot 2$ & $56 \cdot 2$ & $71 \cdot 8^{\star}$ & $31 \cdot 3$ \\
\hline & \pm 21.9 & $\pm 16 \cdot 3$ & $\pm 18 \cdot 9$ & $\pm 15 \cdot 4$ & $\pm 10 \cdot 5$ & \pm 18.0 \\
\hline Trainers & $\begin{array}{r}72 \cdot 3 \\
\pm 17 \cdot 3\end{array}$ & $\begin{aligned} & 100 \cdot 7^{\star} \\
+ & 19 \cdot 3\end{aligned}$ & $\begin{array}{r}44 \cdot 2 \\
+32.7\end{array}$ & $\begin{array}{r}50.2 \\
+12.1\end{array}$ & $\begin{array}{r}76 \cdot 5^{\star} \\
+15.2^{2}\end{array}$ & $\begin{array}{r}58 \cdot 7 \\
+49.2\end{array}$ \\
\hline
\end{tabular}

$\star \mathrm{P}<0.05$

with mean baseline IC's of $89 \%$ predicted showed little or no improvement with ventilatory muscle training. Although the increase in IC was insignificant for the group as a whole, it appears that those quadriplegics with low IC's can improve their IC considerably following inspiratory muscle training.

Only 1 patient experienced an elevation in end-tidal $\mathrm{CO}_{2}$ consistently during the SIP runs with pre-test values ranging from $35-40 \mathrm{~mm} \mathrm{Hg}$ and end SIP test values ranging from $50-58 \mathrm{~mm} \mathrm{Hg}$. This subject complained of no symptoms with this elevation in $\mathrm{CO}_{2}$. The mean $\mathrm{CO}_{2}$ for all other subjects were within normal limits. No ECG abnormalities were observed, and the mean elevation in heart rate at the end of the SIP runs for both groups was 16.2 beats $/ \mathrm{min}$. over the baseline heart rate that day.

Both the trained group and the controls significantly increased MIP and SIP post study (Table III). Although the changes appeared greater in the trainers, the standard deviations were large. The mean pattern of increase in SIP and MIP for the trainers appeared to be progressive over the 8 week study period, while the increases in the control group appear to level off after their fourth testing session (Fig. 2). There were no significant differences in MIP or SIP at any time period between the 2 groups.

Breathing pattern changes observed were the same for both the trainers and the controls (Table IV). Mean frequency was significantly reduced in the control group ( $p<0.05)$, and while the difference was insignificant in the trainers $(\mathrm{p}<0.50)$, quantitatively the decrease in frequency was the same. Mean tidal volume increased slightly in both groups allowing for minute ventilation to remain unchanged. No significant differences in timing were observed between the 2 groups following training, and there were no changes in the variability of any breath component between the 2 groups as assessed by comparing the coefficients of variation.

Only 2 controls and 1 trainer demonstrated any sighs during the pre-training evaluation, and there were no changes in sighing frequency observed posttraining.

As both trainers and controls significantly increased MIP and SIP, the data was pooled, and the effects of increased MIP and SIP on breathing pattern was analysed for the group as a whole. This analysis confirmed the significant decrease in frequency previously observed in the control group only, and found that this was due to a significant increase $(\mathrm{p}<0.05)$ in $\mathrm{Te}(1.91 \pm .43$ to $2.48 \pm \cdot 75$ ). Mean Ti/Ttot for the 12 subjects was $0.405 \pm .049$ pre-test and decreased significantly $(\mathrm{p}<0.05)$ to $0.374 \pm .046$ with the increased MIP and SIP. 


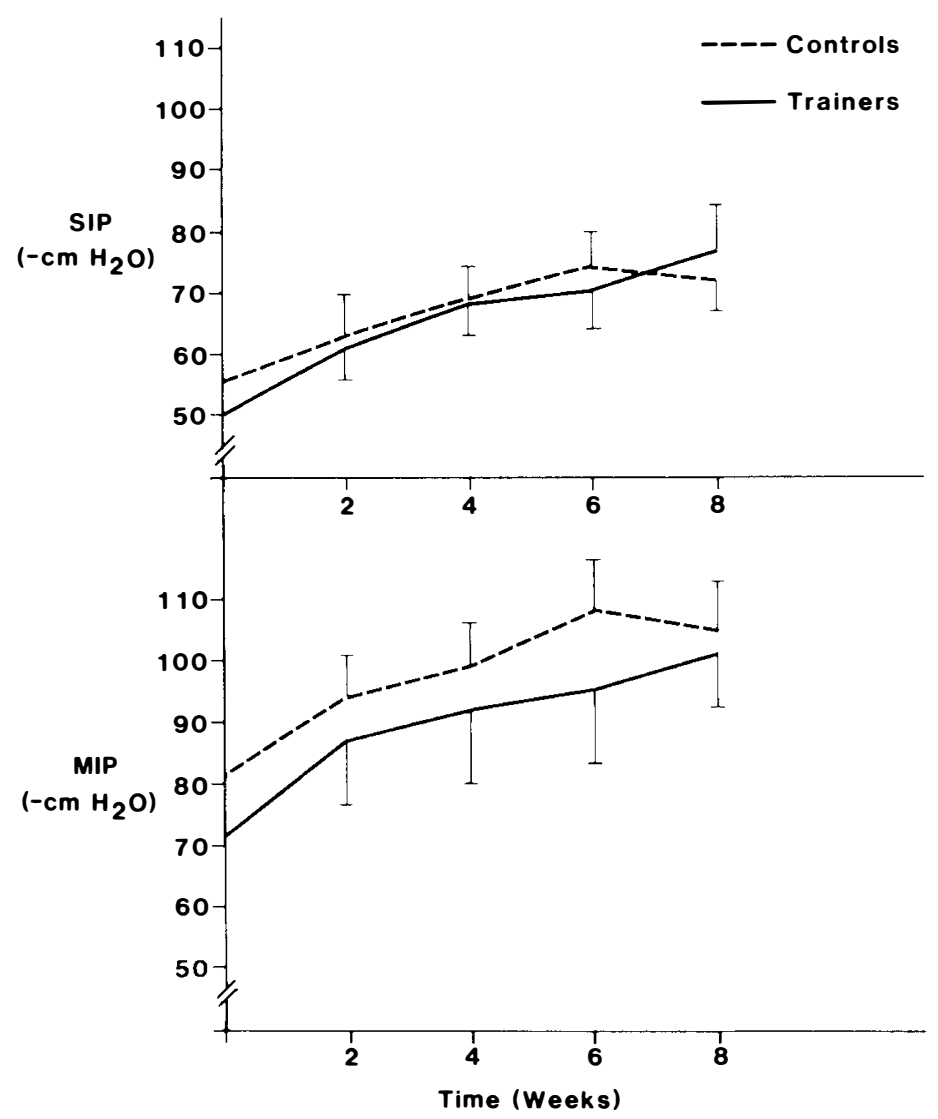

Figure 2 Mean sustainable inspiratory pressures (SIP) and mean maximum inspiratory pressures (MIP) with standard deviation bars for both the trainers (solid line) and the controls (dashed line) at 2 weekly intervals.

\section{Discussion}

The 12 quadriplegics studied showed the same restrictive lung function pattern observed by previous investigators (Stone and Keltz, 1963; McKinley et al., 1969). In spite of this and a rapid shallow breathing pattern, these subjects were able to maintain normal arterial blood gases at rest. This is comparable to the observations in previous studies of chronic quadriplegia (McKinley et al., 1969).

Our inspiratory resistive ventilatory muscle training protocol produced similar increases in muscle strength (MIP) as those observed in training regimes with other quadriplegic patients (Gross et al., 1980); and although endurance was assessed differently in this study as compared to ours, both studies found improved ventilatory muscle endurance with training. Similar improvements in ventilatory muscle strength and endurance following a variety of training protocols has been confirmed in patients with cystic fibrosis (Keens et al., 1977), chronic obstructive pulmonary disease (COPD) (Pardy et al., 1981; Belman and Mittman, 1980), and in normals (Leith and Bradley, 1976). However, our control group also showed significant increases in MIP and SIP. These subjects 
Table IV Timing components of ventilation pre- and post-training

\begin{tabular}{lcccc}
\hline & Trainers & & Controls & \\
& Pre & Post & Pre & Post \\
\hline \# Breaths & 358 & 320 & 380 & 313 \\
& \pm 93 & \pm 91 & \pm 47 & \pm 66 \\
TI & $1 \cdot 42$ & $1 \cdot 50$ & $1 \cdot 21$ & $1 \cdot 36$ \\
& $\pm \cdot 48$ & $\pm \cdot 40$ & $\pm \cdot 30$ & $\pm \cdot 23$ \\
TE & $2 \cdot 00$ & $2 \cdot 47$ & $1 \cdot 82$ & $2 \cdot 49$ \\
& $\pm \cdot 56$ & $\pm \cdot 72$ & $\pm \cdot 27$ & $\pm \cdot 84$ \\
VT & $\cdot 299$ & $\cdot 339$ & $\cdot 325$ & $\cdot 396$ \\
& $\pm \cdot 07$ & $\pm \cdot 08$ & $\pm \cdot 09$ & $\pm \cdot 14$ \\
F & $19 \cdot 5$ & $17 \cdot 4$ & $20 \cdot 2$ & $16 \cdot 9 \star$ \\
& $\pm 5 \cdot 3$ & $3 \cdot 9$ & $\pm 3 \cdot 4$ & $\pm 3 \cdot 1$ \\
VE & $5 \cdot 5$ & $5 \cdot 3$ & $6 \cdot 4$ & $6 \cdot 4$ \\
& $\pm \cdot 77$ & $\pm \cdot 76$ & $\pm 1 \cdot 5$ & $\pm 2 \cdot 0$ \\
VT/TI & $\cdot 223$ & $\cdot 236$ & $\cdot 274$ & $\cdot 302$ \\
& $\pm \cdot 03$ & $\pm \cdot 03$ & $\pm \cdot 07$ & $\pm \cdot 11$ \\
TI/TTOT & $\cdot 417$ & $\cdot 383$ & $\cdot 393$ & $\pm \cdot 06$ \\
& $\pm \cdot 04$ & $\pm \cdot 03$ & $\pm \cdot 06$ & \\
\hline
\end{tabular}

$\star^{\star} \mathrm{p}<0 \cdot 05$

did not participate in any resistive training sessions, except for the stimulus of the testing procedures which was repeated every 2 weeks. Enhanced endurance has been observed in repeated testing in COPD subjects (Richardson et al., 1988). These authors found that experienced COPD subjects who had previously performed an endurance test similar to that described by Knickerson and Keens (1982) on 6 occasions could breathe against a significantly higher maximum baseline load during a 2 minute incremental endurance test than could naive COPD patients. Repeated endurance testing at weekly intervals in these experienced COPD patients, however, did not show a progressive improvement in endurance beyond the baseline values. The COPD study also found no difference in strength (MIP) measurements between naive and experienced COPD subjects, and no improvement in MIP measurements on 3 separate tests conducted 1 week apart. Knickerson and Keens (1982) noted a slight improvement in both MIP and SIP on the second testing of normal subjects but found no additional increases after that time period. Clanton et al. (1985a) found no significant differences in MIP or endurance in normal control subjects who exercised at a subthreshold stimulus $(10 \% \mathrm{MIP})$, in contrast to a normal training group who exercised at a higher stimulus which produced training effects.

While learning could certainly account for the improvement in MIP and SIP between the first and second testing sessions in our study, previous authors experience (Clanton et al., 1985a; Nickerson and Keens, 1982; Richardson et al., 1988) cannot explain the progressive improvement observed beyond this point in both the control and trainer groups (Fig. 2). The testing procedure appears to have induced a similar response in both groups.

The improvement in MIP and SIP observed in previous studies following ventilatory muscle training has been attributed to either alterations in the ventilatory muscles themselves as a result of training, or to an altered strategy of breathing. As breathing pattern in the controls improved similarly to the trainers, this suggests that the testing stimuli delivered at 2 weekly intervals was sufficient to induce a 'training effect'. It is unlikely that these changes reflect a 
training response in the ventilatory muscles themselves as it has been suggested that the ventilatory muscles respond to training in a similar manner to other skeletal muscles (Belman and Sieck, 1982). While, the minimal intensity, frequency and duration of training required to induce training effects in the ventilatory muscles, particularily in quadriplegics is unknown, a training regime consisting of one session every 2 weeks has never been shown to induce training effects in other skeletal muscles. It is more likely that the rigorous testing procedures using a threshold load device resulted in the controls altering their strategy to breathe in a similar manner as the trainers. This altered strategy appears to be able to be maintained, and improved with infrequent reinforcement, and appears to induce changes in resting breathing pattern. Belman et al. (1986) has previously reported that the strategy of breathing in COPD patients during inspiratory resistive breathing significantly alters the patient's ability to sustain a higher load. Previous authors have also observed that ventilatory muscle endurance in normal subjects increased when inspiratory flow was decreased, despite a nearly constant pressure load and duty cycle (Clanton et al., 1985b). Such an altered breathing strategy could account for the enhanced performance during ventilatory muscle endurance testing.

The fact that there was such variability in the improvement in MIP and SIP in the training group could be explained at least in part from the training protocol chosen. The flow rate could be altered with the resistive training device employed, and the pressure generated is dependent on flow. The trainers were allowed to set their own flow rates during training and it is possible that some of them decreased their flow rates sufficiently during the training sessions that they were no longer generating a pressure sufficient to elicit an additional training effect. However, the training pressure was selected assuming a flow rate of approximately 0.3 litres/sec. \pm .05 litres and all trainers were exercising on orifices of $2.5 \mathrm{~mm}$ or less during the last 2 weeks of their training. It has been previously suggested that COPD patients may alter their strategy to breathe against inspiratory resistances and improve performance in ventilatory muscle testing without inducing training effects on the ventilatory muscles themselves (Belman et al., 1986). This could explain the large variation in both the improvements in MIP and SIP, and in breathing pattern alterations observed in our training group. The addition of threshold load training, rather than a flow-dependent resistive device may have more conclusively addressed the question of whether there would be an additive effect of training to that observed by testing alone. A larger sample size would also have assisted, but large groups of quadriplegics with similar lesion levels who would meet the criteria for inclusion in such a study are difficult to find without conducting a multicentre trial. Finally, it is possible that the additional training is unnecessary, as a less frequent stimulus is all that is required to induce an altered strategy to breathe in quadriplegics.

While altering one's strategy to breathe may account for an improvement in test values, it does not explain the changes in resting breathing that were observed. Similar changes in resting breathing pattern were observed in both controls and trainers. Frequency decreased significantly in the control group and similarity, but insignificantly in the trainers. Mean tidal volume increased $18 \%$ of baseline $\mathrm{Vt}$ in the controls and $17 \%$ baseline $\mathrm{Vt}$ in the trainers. This slight 
increase in Vt coupled with a decreased frequency resulted in an unchanged minute ventilation. The tendency towards a slower, deeper breathing pattern following increases in MIP and SIP is quite apparent. Such changes could account for the subjective improvements noted by previous authors in quadriplegics following a ventilatory muscle training regime (Gross et al., 1980). Breathing patterns, and the timing components of the spirogram have not been previously examined following ventilatory muscle training. The pre-study mean $\mathrm{Ti} / \mathrm{T}$ tot for both trainers and controls was $0.405 \pm 0 \cdot 49$. It has been previously suggested that such long Ti/Ttot's predispose individuals to inspiratory muscle fatigue (Bellemare and Grassino, 1983). Following the stimulus of the threshold load testing sessions mean $\mathrm{Ti} / \mathrm{T}$ tot for the combined groups was reduced to $0.374 \pm 046$. This post study $\mathrm{Ti} / \mathrm{T}$ tot is comparable to values that we observed in normal individuals in a previous study (Loveridge et al., 1984b). A shorter $\mathrm{Ti} / \mathrm{T}$ tot is thought to allow for recovery of the diaphragm during expiration and therefore minimise fatigue (Bellemare and Grassino, 1983). Increasing MIP and SIP appears to have resulted in a decrease in $\mathrm{Ti} / \mathrm{T}$ tot towards normal values.

\section{Conclusions}

The repeated presentation of an inspiratory threshold load on the respiratory muscles of quadriplegics appears to induce positive changes in ventilation. These changes likely represent an altered strategy to breathe, rather than any training effects in the ventilatory muscles themselves. This altered strategy to breathe against a resistive load appears to induce an effect on the inspiratory timing mechanism resulting in a shorter $\mathrm{Ti} / \mathrm{T}$ tot, and a slower, deeper respiration during resting ventilation. Whether or not these alterations in resting breathing pattern can be sustained, or whether further threshold loaded inspiratory muscle training can produce greater improvements in breathing remains unclear.

\section{Acknowledgement}

Supported by a Grant from the Sellers Foundation of Manitoba.

\section{References}

Bellemare F, Grassino A 1983 Force reserve of the diaphragm in patients with chronic obstructive pulmonary disease. Fournal of Applied Physiology 55(1):8-15.

Belman MJ, Mittman C 1980 Ventilatory muscle training improves exercise capacity in chronic obstructive pulmonary disease patients. American Review of Respiratory Disease 12:273-280.

Belman MJ, SiECK GC 1982 The ventilatory muscles: Fatigue, Endurance and Training. Chest 82(6):761-765.

Belman MJ, Thomas SG, Lewis MI 1986 Resistive breathing training in patients with chronic obstructive pulmonary disease. Chest 90(5):662-669.

BERGOFSKY EH 1964 Mechanism for respiratory insufficiency after cervical cord injury. Annals of Internal Medicine 61(3):435-447.

BLACK LF, HYATT RE 1971 Maximal static respiratory pressures in generalized neuromuscular disease. American Review of Respiratory Disease 103:641-650.

Clanton TL, Dixon G, Drake J, et al. 1985a Inspiratory muscle conditioning using a threshold loading device. Chest 87(1):62-66.

Clanton TL, Dixon GF, Drake J, et al. 1985b Effects of breathing pattern on inspiratory muscle endurance in humans. Fournal of Applied Physiology 59(6):1834-1841. 
Grassino A, Gross D, MACKLEM PT, et al. 1979 Inspiratory muscle fatigue as a factor limiting exercise. Bulletin Europeen de Physiopathologie Respiratoire 15:105-111.

Gross D, LADD HW, RILEY EJ, et al. 1980 The effect of training on strength and endurance of the diapragm in quadriplegia. American fournal of Medicine 68:27-33.

KeENS TG, KRASTIN IRB, WANNAMAKer EM, et al. 1977 Ventilatory muscle endurance training in normal subjects and patients with cystic fibrosis. American Review of Respiratory Disease 116:853-860.

LEITH DL, BRADLEY M 1976 Ventilatory muscle strength and endurance training. fournal of Applied Physiology 4:508-516.

LOVERIDGE B, WEST P, ANTHONISEN NR, et al. 1983 Single position calibration of the respiratory inductance plethysmograph. Fournal of Applied Physiology 55(3):1031-1034.

Loveridge B, Perez-Padilla R, West P, et al. 1984a Comparison of the stability of the respiratory inductance plethysmograph versus mercury strain gauges in measuring ventilation American Review of Respiratory Disease 129(4): Supplement A82.

LOVERIDGE B, WEST P, ANTHONISEN NR, et al. 1984 Breathing patterns in patients with chronic obstructive pulmonary disease. American Review of Respiratory Disease 130:730-733.

MCKinley AC, Auchincloss JH, Gilbert R, et al. 1969 Pulmonary function, ventilatory control, and respiratory complications in quadriplegic subjects. American Review of Respiratory Disease 100:526-532.

NiCKERSON BG, KeENS TG 1982 Measuring ventilatory muscle endurance in humans as sustainable inspiratory pressure. Fournal of Applied Physiology 52(3):768-772.

PARDY RL, RIVINGTON RN, DesPas PJ, et al. 1981 Inspiratory muscle training compared with Physiotherapy in patients with chronic airflow limitation. American Review of Respiratory Disease 123:421-425.

RICHARDSON J, REID D, DUNn L, et al. 1988 Reproducibility of tests of respiratory muscle performance in chronic obstructive pulmonary disease (COPD). Physiotherapy Canada 40(1):34-39.

STONE DJ, KELTZ H 1963 The effect of respiratory muscle dysfunction on pulmonary function. American Review of Respiratory Disease 88:621-629. 